\title{
Adolescents Sample More Information Prior to Decisions than Adults when Effort Costs Increase
}

Jesse C. Niebaum¹, Anne-Wil Kramer², Hilde M. Huizenga ${ }^{2,3,4}$, \& Wouter van den Bos²

${ }^{1}$ Department of Psychology and Center for Mind and Brain, University of California-

Davis, Davis, California, United States of America

${ }^{2}$ Department of Psychology, University of Amsterdam, Amsterdam, The Netherlands

${ }^{3}$ Amsterdam Brain and Cognition Center, University of Amsterdam, Amsterdam, The

Netherlands

${ }^{4}$ Research Priority Area Yield, University of Amsterdam, Amsterdam, The Netherlands

${ }^{*}$ Correspondence: icniebaum@ucdavis.edu

This is a preprint of a manuscript currently under review. More information about this project, including the preregistration, data, and analytic code, can be found on the project's Open Science Framework Page (osf.io/yqdfe/).

\section{Version Date: June 10, 2021}

\section{Acknowledgements}

We thank Tinka Beemsterboer for organizing adult data collection, Esmee Ris, Myrna Westerveld, Valeria van Thoor, Iris Wierdsma, and Lianne van Schaik for assisting in adolescent data collection, Tristan Konolige for assistance in programming, Ryan Guild for helpful discussion, as well as the participating schools.

\section{Funding}

JCN is supported by the National Science Foundation Graduate Research Fellowship Program (Grant No. 1650042) and an international travel allowance through Graduate Research Opportunities Worldwide. W.v.d.B. is supported by the Jacobs Foundation, European Research Council grant (ERC-2018-StG-803338), Netherlands Organization for Scientific Research grant (NWO-VIDI 016.Vidi.185.068), and a H2020 Consortium on Digital Maturity (DIGYMATEX, H2020 Agreement No. 870578). AK was supported by the Start Impulse grant to NeuroLabNL from the Dutch National Science Agenda (Grant No. 400.17.602). 


\section{Abstract}

Adolescence is marked by increased agency in decision-making and an accompanying increase in risky and impulsive decision-making. Making better decisions typically requires obtaining information relevant to that decision, suggesting that one explanation for increased risky and impulsive behavior in adolescence is a tendency to make illinformed decisions. Adolescents could be especially averse to the effort associated with acquiring information. To investigate this possibility, we recruited adolescents (13-17 years old) in upper-secondary schools and young adults (17-47 years old) attending university in the Netherlands to complete an effort-based information sampling task, in which participants could sample information until obtaining sufficient evidence to make a decision, and effort costs for sampling were systematically varied. Surprisingly, adolescents sampled more evidence than adults before making decisions when sampling effort costs were low; further, adolescents obtained stronger evidence prior to their decisions than adults as effort costs increased, exhibiting less aversion to effort costs associated with information sampling. Computational models supported these findings. Both adolescents and adults used simple heuristics in deciding whether to sample additional information or make a final decision, and adolescents sought a higher evidence threshold before deciding compared with adults. These results suggest that adolescents may require more certainty to make decisions compared with adults and be less averse to effort costs when gathering information to aid decisions.

Keywords: adolescent, decision-making, information sampling, effort costs, heuristics, effort-based decision-making 


\section{Introduction}

Adolescence is marked by increased independence for self-directed decisionmaking and an accompanying increase in risky and impulsive decisions (Crone \& Dahl, 2012; Defoe et al., 2015; Kann et al., 2018; Romer, Reyna, \& Satterthwaite, 2017; Somerville, Jones, \& Casey, 2010; Steinberg, 2007). Many experimental studies have attempted to capture this pattern (for review, see Rosenbaum et al., 2018), focusing on developmental differences in reward sensitivity, peer influence, risk tolerance, and preferences for immediate or future outcomes (Albert, Chein, \& Steinberg, 2013; Blankenstein et al., 2016; Braams et al., 2015; Figner et al., 2009; Lejuez et al., 2003; Mitchell, Schoel, \& Stevens, 2008; van den Bos et al., 2015). In these laboratory tasks, adolescents are typically presented with full information about the consequences of their choices. However, for many decisions associated with risk, such as drug use and sexual activity, and for decisions not stereotypically associated with risk, such as deciding whether or where to attend college, full information is not directly available. Instead, individuals often need to gather information from peers, observation, or other sources prior to making decisions. How, and how much, information is gathered impacts final choices (Rosenbaum et al., 2018; Wulff et al., 2017). However, evidence on developmental differences in information gathering is comparatively scarce.

Some evidence suggests that adolescents gather more information than adults, reflecting a general pattern of decreased exploration from childhood through adulthood (Nussenbaum \& Hartley, 2019; Gopnik et al., 2017). For example, adolescents explore more information than adults when potential choices lead to immediate reward (e.g., Jepma et al., 2020; Kwak et al., 2015). However, this greater exploratory behavior may 
not generalize to contexts in which rewards after information gathering are delayed. When selecting between options offering high reward or high informational value, adolescents were more likely to favor the high reward option than adults (Somerville et al., 2017). Adolescents also sampled less information than older children and adults when drawing from two lotteries before picking which lottery to play for a reward, making decisions with comparatively less information (van den Bos \& Hertwig, 2017). The delay in reward incurred by additional information sampling may discourage adolescents from more sampling (Galván, 2010; Christakou, Brammer, \& Rubia, 2011; de Water, Cillessen, \& Scheres, 2014; van den Bos et al., 2015). Adolescents may also be more confident that the available information is sufficient to make good decisions; even when selecting less-rewarding options than adults, adolescents expressed similar levels of confidence in their decisions (Jepma et al., 2020).

Another underexplored possibility is that adolescents are more averse to the effort costs required to obtain information. Although some evidence suggests that adolescents are less sensitive to effort costs than adults when exerting effort for reward (Rodman et al., 2020; Sullivan-Toole et al., 2019), effort in these paradigms also led to immediate rewards. Thus, adolescents may be more averse than adults to effort costs when not directly associated with immediate reward. Exerting effort to accumulate information for decisions without immediate payoffs may be particularly aversive for adolescents.

We investigated whether adolescents sample less information than adults prior to decisions and whether adolescents are more sensitive than adults to sampling effort costs. To this end, we developed an effort-based information sampling task (adapted 
from Clark et al., 2006, 2009). Sampling effort costs were manipulated by varying the number of mouse clicks to sample information. In this task, participants see 25 gray squares that can be clicked to reveal one of two colors underneath. Participants are instructed select which color is the underlying majority and can decide which color is the majority at any point during the trial. Several task features mitigate confounding factors on effort-based sampling decisions. First, the effort required to sample information was made explicit, preventing differences in individuals' ability to learn about implicit effort costs from influencing decisions; second, no feedback was provided to avoid strategy formation and prevent reward and punishment sensitivities from affecting subsequent decisions; third, sampled information was always available, reducing working memory demands, which influence information sampling (Hertwig et al., 2004); and lastly, certainty could always be achieved if desired by sampling until a majority is flipped (e.g., 13 squares of one color).

Given prior evidence of adolescents' limited information sampling prior to decisions and decreased preference for information over reward compared with adults, we predicted less information sampling in adolescents than young adults.

Consequently, we also predicted that age-related differences in sampling would become more pronounced as the effort costs to sample information increased, such that adolescents would decrease sampling more than adults if effort costs for sampling increased. Additionally, Bayesian models from prior research and novel heuristic models were fit to better determine the mechanisms underlying differences in information sampling behavior (Hauser et al., 2017). 


\section{Transparency and Openness}

This study was preregistered and was performed according to the preregistration except where explicitly indicated otherwise. All data, materials, and analysis code, including the preregistration, are publicly available on the project's repository on the Open Science Framework (View only link for peer review: https://osf.io/yqdfe/?view_only=57d0b8736d714283a1f74eba24cc4ed5). All analyses were conducted in R Studio, version 1.2.5042 (RStudio Team, 2020). Mixed models were run using the ImerTest package (Kuznetsova, Brockhoff, \& Christensen, 2015), built upon. The snowfall package was used for conducting computational models (Knaus et al., 2009). Data were visualized using the ggplot2 and ggpubr packages (Kassambra \& Kassambra, 2020; Wickham, 2011).

\section{Participant Sample}

Adults $(\mathrm{N}=359 ; 20.53$ years $(\mathrm{SD}=2.34$; range: $17.83-47.00) ; 247$ female, 2 nonbinary, 11 unreported) were recruited from the local university participant pool and completed the protocol for partial course credit. Adolescents $(\mathrm{N}=95 ; 15.02$ years $(S D=0.52$; range: 13.59-17.00); 48 female, 1 unreported) were recruited from secondary schools in The Netherlands. We only included adolescents attending upperlevel secondary school students because only these students typically continue to university, increasing the validity of age comparisons. Participating adolescents provided informed consent and parental passive or active consent, depending on school preference. All procedures were approved by the local Ethics Review Board. Deviating from the pre-registration, we decided prior to analyses to eliminate participants who failed to sample any square on all medium- or high-effort trials, reasoning that these 
participants did not experience these sampling effort costs. This criterion excluded a similar percentage of participants across groups: 28 adults (6.1\%) and 6 adolescents (5.9\%). Highly convergent results were obtained with the full sample (Supplementary Material).

\section{Procedure}

The study took place over one session in which the effort-based information sampling task was completed amongst other cognitive assessments and questionnaires. The full protocol typically ranged from 50-80 minutes. Adolescents completed the session in groups of $15-28$ participants. Adults completed the session in two groups of approximately 190 participants in a large study hall. The information sampling task was administered using NeuroTask (scripting.neurotask.com; Murre, 2016), an online task environment. Participants also completed the Intolerance of Uncertainty Scale (Carleton, Norton, \& Asmundson, 2007), administered via Qualtrics (Provo, Utah). Analyses of the latter data are in Supplementary Material.

\section{Effort-based Information Sampling Task}

The effort-based information sampling task was adapted from Clark and colleagues (2009), with several alterations (Figure 1). On each trial, participants saw a $5 \times 5$ array of gray squares that could be flipped via clicking to reveal one of two colors underneath, yellow or blue. Participants were instructed to decide which color was the underlying majority color. Participants selected the majority color by clicking one of two buttons beneath the array at any point during the trial. Sampled squares remained flipped for the entire trial. To manipulate sampling effort costs, participants were instructed that the number of clicks needed to flip a square would vary across trials (1, 
4, or 12 clicks), similar to other effort-based decision-making tasks (Treadway et al., 2009; Sullivan-Toole et al., 2019). A small square in the upper right corner indicated how many clicks were needed to sample a square. To discourage participants from sampling less only to proceed through the task more quickly, the instructions indicated that participants would play for 15 minutes, thereby mitigating potential confounding opportunity costs. Instructions emphasized that participants could sample as many squares as desired until feeling certain enough to make a decision. Participants completed 10 trials at each effort level, resulting in 30 trials, and trial order was randomized within participants. The same 10 screen arrangements of blue and yellow squares were used at each effort level. The majority color on each screen ranged from 17 to 20 out of the 25 squares. A 2-second interval was used between trials, and no feedback was provided. If participants had played for more than 15 minutes, the task ended after the current trial. Participants finishing earlier progressed in the study protocol or were dismissed (if no task followed the current one). Instead of the preregistered procedure of rewarding participants on two randomly selected trials per effort level, every participant was compensated $€ 1.00$ after the full protocol due to practical concerns in compensating participants different amounts while testing in groups. Adolescent instructions were translated into Dutch and reviewed by three native speakers (Supplementary Material).

\section{Analyses}

Two primary outcome variables were calculated for each trial and then averaged over trials at each difficulty level within participants: total squares sampled (Clark et al., 2009) and $p$ (correct), that is, the probability that the color chosen is the majority color, 
which was formalized as a Bayesian inference problem using an uninformative prior updated with the sampled information at the time of decision, as follows (Bennett et al., 2017):

$$
P(\text { correct })=\operatorname{Pr}\left(\theta \geq 13 \mid n_{1}, n_{2}\right)=\sum_{M=13}^{25} \operatorname{Pr}\left(\theta=M \mid n_{1}, n_{2}\right)
$$

where $n_{1}$ is the number of squares flipped of the selected majority color, $n_{2}$ is the number of squares flipped of the color not selected, and $\operatorname{Pr}\left(\theta \geq 13 \mid n_{1}, n_{2}\right)$ is the likelihood of the sampled boxes if the true number of boxes of the chosen color $(\theta)$ were the majority $(M)$ (remember that the total number of boxes is 25$)$. $\mathrm{p}$ (correct) provides complementary information to the number of squares sampled, as participants could see different strengths of evidence while sampling the same number of squares (e.g., 12 blue and 4 yellow squares provide stronger evidence for blue (>99\%) than 8 blue and 8 yellow squares (50\%)). Analysis of discrimination errors, in which participants do not select the shown majority color (Clark et al., 2009), is included in Supplementary Materials. Mixed model linear regressions were conducted with random slopes (effect of effort cost) and intercepts for participants, including covariance between random slopes and intercepts. Low-effort trials were coded as 0 to directly compare group differences with minimal effort costs, medium-effort trials as 1 , and high-effort trials as 2 . Adolescents were coded as -.5 , and adults as .5. Thus, main effects indicate age differences on low-effort trials, and interactions indicate whether adolescents and adults differ in the effect of effort costs.

\section{Computational Modeling}


To better understand the mechanisms underlying information sampling, we fitted several computational models. We fitted two families of models: regular Bayesian (Hauser et al., 2017; Ma, Sanfey, \& Ma, 2020) and new heuristic models. We describe the motivation for these models below, full mathematical descriptions of the models are included in the Supplemental Materials. The Bayesian models consist of prior beliefs over the distribution of yellow and blue squares and an evolving posterior distribution. Given that participants initially have no information about the distribution, the prior is an uninformative beta distribution, and the posterior is updated with each sample.

The first Bayesian model (Bayesian Optimal) computes the value of both sampling more and the value of stopping at each possible state (combination of blue and yellow boxes flipped) a participant can be in. When 13 boxes of one color are flipped, the majority color is known with full certainty. Thus, these states (13 blue or yellow) are considered end states, where sampling more has no value (zero) and the expected value of stopping is the reward for being correct (one). By starting with the final state, we can retrogress in sampling time to obtain the expected value of sampling or stopping for every possible state. The value of stopping is the product of the probability that a color is the majority and a one point reward for accuracy. The value of sampling is the product of the probability of the next state (i.e., state with one more yellow or one more blue square) and the expected value of that next state. The probability of the next state is determined by the current state. For example, if the current sample consist of a lot more blue than yellow squares, it is also more likely that the next square will be blue. The value of the next state is again the expected value of stopping and value of sampling more. This process proceeds until one of the end states 
is met, and therefore, these end states can be used to calculate the expected value of each state by recursively taking steps back in time (i.e., dynamic programming). Note that in principle more information will always yield better predictions of the majority, and the optimal solution is to sample until 13 squares of one color are revealed. However, this Bayesian models can be adapted to accommodate that sampling itself is costly. In our models, the sampling cost is a free parameter that reflects subjective sampling cost. In sum, this model calculates the expected value of sampling for more information by calculating how much further sampling would improve a future decision minus the cost of search (see Supplemental Material).

The second Bayesian model (Bayesian Uncertainty) also assume that agents use Bayesian updating of the beta distribution and that search is costly, but now sampling stops when a subjective certainty criterion is met. The uncertainty of a state at a given time is determined by the standard deviation (SD) of the beta distribution, and the model stops sampling when the SD is below a threshold. In this model both the threshold and the cost of search are free parameters.

Finally, we also added versions of the Bayesian models in which the cost of sampling with a trial increases over time (urgency), indicating increasing impatience to make a decision regardless of the available evidence with each box opened. Following Hauser et al. (2017), the urgency parameter was fixed over participants and resulted in a trial by trial increase in the subjective search cost.

The heuristic models are based on simple count data from the total squares sampled. The absolute difference model (Absolute Difference) assumes that agents only attend to the absolute difference between yellow and blue squares and stop 
sampling when a threshold of minimum difference is met. Another heuristic model (Total Squares) assumes that agents stop sampling after a threshold of a number of sampled squares is met. Finally, we implemented a heuristic model that uses the combination of absolute difference and total squares sampled (Two-rule). This model continues to sample until a threshold of a number of sampled squares is met or until a difference threshold is hit, with no priority over each rule. For all these heuristic models, the threshold value(s) were free parameters estimated for each participant.

\section{Model Fitting}

The free parameters of the models were estimated by fitting the model predictions to participants' decisions, we did so per participant, per effort level. For model selection purposes, we computed and then summed the Bayesian information criterion (BIC) across all subjects, we did so for all models, where lower BIC values indicate better fit. All model parameters were estimated using the L-BFGS-B method in the optim toolbox in R (Byrd et al., 1995). This algorithm allows box constraints in which each parameter can be given a lower and/or upper bound (Supplementary Materials). To prevent local minima, we generated 20 sets of randomly generated starting values for the parameter values from a uniform distribution within the bounds. Models were fit to each effort level separately to determine how effort impacted sampling strategies. Parameters of the best-fitting model were used to study developmental differences in sampling strategies.

\section{Results}

Sampling effort is higher in adolescents than adults 
Descriptive statistics for outcome variables are included in Table 1. The number of squares flipped decreased with increasing effort costs $\left(\beta=-2.83, t_{(451.98)}=-23.90\right.$, $p<.001)$. In contrast to our hypotheses, adolescents sampled more squares on low-effort trials than adults $\left(\beta=-1.37, t_{(451.98)=-2.35,} p=.019\right)$, and no significant interaction between age group and effort level was observed $\left(\beta=-0.32, t_{(451.98)}=-1.36, p=.176\right)$. Follow-up $t-$ tests showed that adolescents sampled more squares than adults at all effort levels (all ps $<.05)$ (Figure 2A). $p$ (correct) decreased with increasing effort $(\beta=-0.04, t(452.00)=-14.31$,

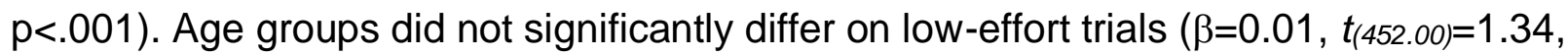
$p=.183$ ); however, a significant group by effort level interaction was observed $(\beta=-0.02$, $t_{(452.00)=-3.53, p<.001)}$, showing that adults decreased more in $p$ (correct) than adolescents as effort costs increased (Figure 2B). Follow-up t-tests indicated that adults and adolescents did not significantly differ in $\mathrm{p}$ (correct) on the low- and medium-effort levels ( $p s>.3$ ) but that $p$ (correct) was significantly higher in adolescents than adults on high-effort trials $\left(t_{(153.44)=3.05, p=.019)}\right.$. These results indicate that adolescents and adults had similar probability of responding correctly on low-effort trials, but adolescents had a higher probability of responding correctly on high effort trials.

Trials without Information Sampling Indicate Effort Avoidance

During data collection, we discovered that participants completed some trials without any sampling. We subsequently explored whether no-sample trials differed by age and by effort level using the full data sample. No-sample trials increased with effort level $\left(\beta=2.43, t_{452.00)}=4.83, p<.001\right)$ and were less frequent in adults than adolescents on low-effort trials $\left(\beta=-2.33, t_{(452.00)}=-2.16, p=.031\right)$. A significant group by effort level 
interaction was observed $\left(\beta=1.99, t_{(452.00)}=1.98, p=.048\right)$, indicating that adults selectively avoided high-effort trials more than adolescents.

Decision Accuracy is Similar Across Groups

We explored decision accuracy to examine whether the additional sampling by adolescents led to better task performance. Accuracy was high in both groups at all effort levels (Adults: 97\%, 95\%, and 90\%; Adolescents: 96\%, 93\%, and 93\%, for low-, medium-, and high-effort trials, respectively). Accuracy decreased with increased effort level $\left(\beta=-0.03, t_{(451.96)=-7.29,}<<.001\right)$ and was higher in adults than adolescents on loweffort trials $\left(\beta=0.02, t_{(451.77)}=2.49, p=.013\right)$. A group by effort level interaction was observed $\left(\beta=-0.02, t_{(451.96)}=-2.58, p=.010\right)$, indicating that adults had higher accuracy on low-effort trials but that accuracy decreased more as effort costs increased compared with adolescents. When excluding no-sample trials, accuracy decreased with increased effort levels $\left(\beta=-0.02, t_{(491.16)}=-5.42, p<.001\right)$, but no significant effect of age group $\left(\beta=0.01, t_{(849.18)}=1.36, p=.174\right)$ or interaction was observed $\left(\beta=-0.01, t_{(491.16)}=-1.57\right.$, $p=.118$ ), suggesting that adolescents' additional sampling effort did not greatly improve decision accuracy.

Computational model comparison

BIC's are given in Table 2. Model comparison revealed that the heuristic models generally outperformed the Bayesian family of models, suggesting that participants of all ages used simple rules for deciding when to stop sampling. The Two-rule heuristic model best described behavior. These results suggest that participants generally sampled until they met an evidence threshold (overall kdiff: 8.31 (SE=0.16)) or stopped when they flipped a fixed number of squares (overall $k_{\text {tot: }} 14.29(\mathrm{SE}=0.18)$ ). Simulations 
based on the best fitting parameters for each individual revealed that the model predicts the reduction in sampling with increasing sampling cost and captures individuals with different sampling levels well (Supplementary Materials).

Increased evidence thresholds for decisions in adolescents

Finally, in the Two-rule model, we tested for developmental differences in the two threshold values using mixed model regression with random intercepts and slopes for participants across effort levels. The total squares flipped threshold $\left(k_{\text {tot }}\right)$ decreased with increasing effort costs $\left(\beta=-3.31, t_{4452.00)=-17.28,} p<.001\right.$; Figure 3$)$. As expected, people made decisions after sampling fewer squares on high effort trials, regardless of the evidence. No main group effect was observed $\left(\beta=-0.50, t_{(452.00)=-0.75,} p=.45\right)$; however, a significant group by effort level interaction was observed $\left(\beta=-1.50, t_{(452.00)=-3.91}\right.$, $p<.001)$, suggesting adolescents sampled more than adults as effect costs increased. The absolute color difference threshold ( $\left.k_{\text {diff }}\right)$ also decreased with effort costs $(\beta=-2.24$, $t(452.00)=-11.63, p<.001 ;$ Figure 3$)$. A main group effect was observed $\left(\beta=-2.17, t_{(452.00)=-}\right.$ 3.28, $p<.01)$, and no significant interaction was observed $\left(\beta=-0.36, t_{(452.00)}=-0.93\right.$, $\mathrm{p}=.351$ ), suggesting that adolescents maintained a higher threshold for evidence than adults across effort levels.

\section{Discussion}

Before making important choices, we often have the opportunity to search for information. This information can be used to improve the outcomes of our decisions but requires some effort to obtain. Given prior evidence of adolescents' increased impulsivity and decreased preference for information over reward compared with adults, we predicted less information sampling in adolescents than young adults. 
Consequently, we also predicted that age-related differences in sampling would become more pronounced with increased effort costs, such that adolescents would decrease sampling more than adults if effort costs for sampling information increased.

Adolescents and adults were sensitive to sampling effort costs. Both groups decreased information sampling and obtained weaker evidence for decisions as effort costs increased. Surprisingly, however, adolescents sampled more information than adults prior to making decisions and were more willing than adults to expend effort to sample information as effort costs increased. We applied computational modeling to further investigate the possible mechanism underlying these developmental effects. These analyses revealed that a heuristic two-threshold model, which combined a minimum evidence threshold for deciding and an absolute sampling threshold, outperformed the more sophisticated Bayesian models in predicting adolescent and adult sampling behavior (Hauser et al., 2017; Ma et al., 2020), suggesting that participants relied on a simple strategy when sampling information to make decisions. Differences between age groups in the heuristic model were largely driven by adolescents exhibiting higher evidence thresholds for making decisions. Evidence thresholds decreased linearly with increasing effort costs in adults, whereas sampling thresholds were more similar on medium- and high-effort trials in adolescents, indicating that adolescents maintained a minimum level of evidence for making decisions even as effort costs increased.

The increased information sampling in adolescents may seem at odds with prior work showing that adolescents sampled less from different lotteries prior to choosing which lottery to play than children and adults (van den Bos \& Hertwig, 2017) and 
showed less aversion to ambiguity than adults (Blankenstein et al,. 2016). However, several theoretically meaningful task differences may contribute to these divergent results. First, our sampling task differs from previous paradigms in that certainty can be achieved with sufficient sampling, whereas in prior studies, uncertainty was irreducible (see Rosenbaum \& Hartley, 2019). The irreducible uncertainty in other sampling tasks, paired with a more positive attitude towards ambiguity (van den Bos \& Hertwig, 2017), may have led to the reduced sampling in adolescents in previous paradigms. Second, immediate rewards were not available in our paradigm. Because adolescents show greater impulsivity and heightened sensitivity to rewards and punishment compared with older children and adults (Albert \& Steinberg, 2011; Shulman et al., 2016; Spear, 2011; van Duijvenvoorde et al., 2010), they could sample less information at this age when immediate rewards are available.

Adolescents were also more willing to put forth the effort to sample information than adults at higher effort costs, indicating that adolescents may be more willing to expend extra effort to gain relevant information for their decisions. These results are in line with recent evidence showing that adolescents were less averse to higher effort costs to obtain reward and persisted in exerting effort longer than adults even after earning rewards (Sullivan-Toole et al., 2019; Rodman et al., 2020). Similar to this prior work, in which adolescents' extra sampling effort did not yield higher rewards than adults, greater sampling within increasing effort costs here did not result in appreciably higher task accuracy compared with adults. Collectively, these results suggest that adults better conserve and calibrate their effort by not sampling more information than needed to maintain good performance. 
Our study has several limitations that potentially constrain its generalizability. Sampling behavior may change outside of the classroom or testing environment. Being informed that the task would take 15 minutes may have diminished the ecological validity our task, as decision horizons are not always fully known in the real-world. Further, our task reduced working memory demands, which may be required in different decision-making contexts and has been shown to influence information sampling (Rakow \& Rahim, 2010; van den Bos \& Hertwig, 2017). Moreover, our sample was restricted to academically high-performing individuals in the Netherlands, which increased the validity of our age comparisons but reduces generalizability to other populations.

\section{Conclusions}

The present study introduces an effort-based information sampling task that enables explicit manipulation of sampling effort costs and assessments for how much evidence individuals acquire prior to making decisions. Effort-based decision-making remains relatively underexplored across development. Unexpectedly, adolescents sampled more information than adults, requiring a higher threshold of evidence, prior to making decisions. Further, adolescents continued to sample more information than adults even as effort costs increased, suggesting that adolescents may be less averse to effort costs. The additional sampling observed in adolescents did not result in substantial improvements in task performance, indicating that the calibration of sampling effort for decision accuracy continues to improve into adulthood.

Computational models indicated that both adolescents and adults relied on simple sampling strategies, making decisions once reaching a certain evidence threshold or 
after sampling a certain amount. Our findings shed light on the complexity of factors influencing adolescent decision-making, suggesting that risky and impulsive decisions in adolescence may not be due an aversion to effort costs in gaining information but instead maybe due to other factors, such as reward sensitivity, impulsivity, and improvements in working memory. 


\section{References}

Albert, D., Chein, J., \& Steinberg, L. (2013). The teenage brain: Peer influences on adolescent decision making. Current Directions in Psychological Science, 22(2), 114-120. https://doi.org/10.1177/0963721412471347

Albert, D., \& Steinberg, L. (2011). Judgment and decision making in adolescence. Journal of Research on Adolescence, 21(1), 211-224. https://doi.org/10.1111/i.1532-7795.2010.00724.x

Bennett, D., Oldham, S., Dawson, A., Parkes, L., Murawski, C., \& Yücel, M. (2017). Systematic overestimation of reflection impulsivity in the Information Sampling Task. Biological Psychiatry, 82(4), e29-e30.

\section{https://doi.org/10.1016/j.biopsych.2016.05.027}

Blankenstein, N. E., Crone, E. A., van den Bos, W., \& van Duijvenvoorde, A. C. (2016). Dealing with uncertainty: Testing risk-and ambiguity-attitude across adolescence. Developmental Neuropsychology, 41(1-2), 77-92. https://doi.org/10.1080/87565641.2016.1158265

Braams, B. R., van Duijvenvoorde, A. C., Peper, J. S., \& Crone, E. A. (2015). Longitudinal changes in adolescent risk-taking: a comprehensive study of neural responses to rewards, pubertal development, and risk-taking behavior. Journal of Neuroscience, 35(18), 7226-7238. https://doi.org/10.1523/JNEUROSCI.476414.2015

Byrd, R. H., Lu, P., Nocedal, J., \& Zhu, C. (1995). A limited memory algorithm for bound constrained optimization. SIAM Journal on scientific computing, 16(5), 11901208. https://doi.org/10.1137/0916069

Carleton, R. N., Norton, M. P. J., \& Asmundson, G. J. (2007). Fearing the unknown: A short version of the Intolerance of Uncertainty Scale. Journal of Anxiety Disorders, 21(1), 105-117. https://doi.org/10.1016/j.janxdis.2006.03.014

Christakou, A., Brammer, M., \& Rubia, K. (2011). Maturation of limbic corticostriatal activation and connectivity associated with developmental changes in temporal discounting. Neuroimage, 54(2), 1344-1354. https://doi.org/10.1016/j.neuroimage.2010.08.067 
Churchland, A. K., Kiani, R., \& Shadlen, M. N. (2008). Decision-making with multiple alternatives. Nature Neuroscience, 11(6), 693-702. https://doi.org/10.1038/nn.2123

Clark, L., Robbins, T. W., Ersche, K. D., \& Sahakian, B. J. (2006). Reflection impulsivity in current and former substance users. Biological Psychiatry, 60(5), 515-522. https://doi.org/10.1016/j.biopsych.2005.11.007

Clark, L., Roiser, J. P., Robbins, T. W., \& Sahakian, B. J. (2009). Disrupted 'reflection' impulsivity in cannabis users but not current or former ecstasy users. Journal of Psychopharmacology, 23(1), 14-22. https://doi.org/10.1177/0269881108089587

Crone, E. A., \& Dahl, R. E. (2012). Understanding adolescence as a period of socialaffective engagement and goal flexibility. Nature Reviews Neuroscience, 13(9), 636-650. https://doi.org/10.1038/nrn3313

de Water, E., Cillessen, A. H., \& Scheres, A. (2014). Distinct age-related differences in temporal discounting and risk taking in adolescents and young adults. Child Development, 85(5), 1881-1897. https://doi.org/10.1111/cdev.12245

Defoe, I. N., Dubas, J. S., Figner, B., \& Van Aken, M. A. (2015). A meta-analysis on age differences in risky decision making: Adolescents versus children and adults. Psychological Bulletin, 141(1), 48. https://doi.org/10.1037/a0038088

Figner, B., Mackinlay, R. J., Wilkening, F., \& Weber, E. U. (2009). Affective and deliberative processes in risky choice: age differences in risk taking in the Columbia Card Task. Journal of Experimental Psychology: Learning, Memory, and Cognition, 35(3), 709. https://doi.org/10.1037/a0014983

Galván, A. (2010). Adolescent development of the reward system. Frontiers in Human Neuroscience, 4, 6. https://doi.org/10.3389/neuro.09.006.2010

Hauser, T. U., Moutoussis, M., lannaccone, R., Brem, S., Walitza, S., Drechsler, R., ... \& Dolan, R. J. (2017). Increased decision thresholds enhance information gathering performance in juvenile obsessive-compulsive disorder (OCD). PLoS Computational Biology, 13(4), e1005440. https://doi.org/10.1371/journal.pcbi.1005440 
Hertwig, R., Barron, G., Weber, E. U., \& Erev, I. (2004). Decisions from experience and the effect of rare events in risky choice. Psychological science, 15(8), 534-539. https://doi.org/10.1111/j.0956-7976.2004.00715.x

Inzlicht, M., Shenhav, A., \& Olivola, C. Y. (2018). The effort paradox: Effort is both costly and valued. Trends in Cognitive Sciences, 22(4), 337349. https://doi.org/10.1016/.tics.2018.01.007

Jenkins, L., Myerson, J., Hale, S., \& Fry, A. F. (1999). Individual and developmental differences in working memory across the life span. Psychonomic Bulletin \& Review, 6(1), 28-40. https://doi.org/10.3758/BF03210810

Jepma, M., Schaaf, J. V., Visser, I., \& Huizenga, H. M. (2020). Uncertainty-driven regulation of learning and exploration in adolescents: A computational account. PLOS Computational Biology, 16(9), e1008276. https://doi.org/10.1371/journal.pcbi.1008276

Kann, L., McManus, T., Harris, W. A., Shanklin, S. L., Flint, K. H., Queen, B., ... \& Lim, C. (2018). Youth risk behavior surveillance-United States, 2017. MMWR Surveillance Summaries, 67(8), 1. 10.15585/mmwr.ss6708a1

Kassambara, A., \& Kassambara, M. A. (2020). Package 'ggpubr'.

Knaus, J., Porzelius, C., Binder, H., \& Schwarzer, G. (2009). Easier parallel computing in $\mathrm{R}$ with snowfall and sfCluster. The R Journal, 1(1), 54-59.

Kobayashi, K., \& Hsu, M. (2017). Neural mechanisms of updating under reducible and irreducible uncertainty. Journal of Neuroscience, 37(29), 6972-6982. https://doi.org/10.1523/JNEUROSCI.0535-17.2017

Kuznetsova, A., Brockhoff, P. B., \& Christensen, R. H. B. (2015). ImerTest: tests in linear mixed effects models. R package version 2.0-20. Vienna: $R$ Foundation for Statistical Computing. 10.18637/JSS.V082.113

Kwak, Y., Payne, J. W., Cohen, A. L., \& Huettel, S. A. (2015). The rational adolescent: Strategic information processing during decision making revealed by eye tracking. Cognitive Development, 36, 20-30. https://doi.org/10.1016/i.cogdev.2015.08.001

Lejuez, C. W., Aklin, W. M., Zvolensky, M. J., \& Pedulla, C. M. (2003). Evaluation of the Balloon Analogue Risk Task (BART) as a predictor of adolescent real-world risk- 
taking behaviours. Journal of Adolescence, 26(4), 475-479.

https://doi.org/10.1016/S0140-1971(03)00036-8

Ma, I., Sanfey, A. G., \& Ma, W. J. (2020). The social cost of gathering information for trust decisions. Scientific reports, 10(1), 1-9. https://doi.org/10.1038/s41598-02069766-6

Mitchell, S. H., Schoel, C., \& Stevens, A. A. (2008). Mechanisms underlying heightened risk taking in adolescents as compared with adults. Psychonomic bulletin \& review, 15(2), 272-277. https://doi.org/10.3758/PBR.15.2.272

R Core Team. (2020). R: A language and environment for statistical computing (Version 4.0.2). Vienna, Austria: R Foundation for Statistical Computing.

Rakow, T., \& B. Rahim, S. (2010). Developmental insights into experience-based decision making. Journal of Behavioral Decision Making, 23(1), 69-82. https://doi.org/10.1002/bdm.672

Rodman, A. M., Powers, K. E., Insel, C., Kastman, E. K., Kabotyanski, K. E., Stark, A. M., Worthington, S., \& Somerville, L. H. (2021). How adolescents and adults translate motivational value to action: Age-related shifts in strategic physical effort exertion for monetary rewards. Journal of Experimental Psychology: General, 150(1), 103-113. https://doi.org/10.1037/xge0000769

Romer, D., Reyna, V. F., \& Satterthwaite, T. D. (2017). Beyond stereotypes of adolescent risk taking: Placing the adolescent brain in developmental context. Developmental cognitive neuroscience, 27, 19-34. https://doi.org/10.1016/i.dcn.2017.07.007

Rosenbaum, G. M., \& Hartley, C. A. (2019). Developmental perspectives on risky and impulsive choice. Philosophical Transactions of the Royal Society B, 374(1766), 20180133. https://doi.org/10.1098/rstb.2018.0133

Rosenbaum, G. M., Venkatraman, V., Steinberg, L., \& Chein, J. M. (2018). The influences of described and experienced information on adolescent risky decision making. Developmental review, 47, 23-43. https://doi.org/10.1016/j.dr.2017.09.003

Shulman, E. P., Smith, A. R., Silva, K., Icenogle, G., Duell, N., Chein, J., \& Steinberg, L. (2016). The dual systems model: Review, reappraisal, and 
reaffirmation. Developmental cognitive neuroscience, 17, 103-117. https://doi.org/10.1016/i.den.2015.12.010

Somerville, L. H., Jones, R. M., \& Casey, B. J. (2010). A time of change: behavioral and neural correlates of adolescent sensitivity to appetitive and aversive environmental cues. Brain and cognition, 72(1), 124-133. https://doi.org/10.1016/j.bandc.2009.07.003

Somerville, L. H., Sasse, S. F., Garrad, M. C., Drysdale, A. T., Abi Akar, N., Insel, C., \& Wilson, R. C. (2017). Charting the expansion of strategic exploratory behavior during adolescence. Journal of Experimental Psychology: General, 146(2), 155164. https://doi.org/10.1037/xge0000250

Spear, L. P. (2011). Rewards, aversions and affect in adolescence: emerging convergences across laboratory animal and human data. Developmental cognitive neuroscience, 1(4), 390-403. https://doi.org/10.1016/j.dcn.2011.08.001

Steinberg, L. (2007). Risk taking in adolescence: New perspectives from brain and behavioral science. Current directions in psychological science, 16(2), 55-59. https://doi.org/10.1111/j.1467-8721.2007.00475.x

Sullivan-Toole, H., DePasque, S., Holt-Gosselin, B., \& Galván, A. (2019). Worth working for: The influence of effort costs on teens' choices during a novel decision making game. Developmental cognitive neuroscience, 37, 100652. https://doi.org/10.1016/i.den.2019.100652

Treadway, M. T., Buckholtz, J. W., Schwartzman, A. N., Lambert, W. E., \& Zald, D. H. (2009). Worth the 'EEfRT'? The effort expenditure for rewards task as an objective measure of motivation and anhedonia. PloS one, 4(8). https://doi.org/10.1371/journal.pone.0006598

Van Den Bos, W., \& Hertwig, R. (2017). Adolescents display distinctive tolerance to ambiguity and to uncertainty during risky decision making. Scientific reports, 7(1), 1-11. https://doi.org/10.1038/srep40962

Van Den Bos, W., Rodriguez, C. A., Schweitzer, J. B., \& McClure, S. M. (2015). Adolescent impatience decreases with increased frontostriatal connectivity. Proceedings of the National Academy of Sciences, 112(29), E3765E3774. https://doi.org/10.1073/pnas.1423095112 
Van Duijvenvoorde, A. C., Jansen, B. R., Visser, I., \& Huizenga, H. M. (2010). Affective and cognitive decision-making in adolescents. Developmental Neuropsychology, 35(5), 539-554. https://doi.org/10.1080/87565641.2010.494749

Wickham, H. (2011). ggplot2. Wiley Interdisciplinary Reviews: Computational Statistics, 3(2), 180-185.

Wulff, D. U., Mergenthaler-Canseco, M., \& Hertwig, R. (2018). A meta-analytic review of two modes of learning and the description-experience gap. Psychological Bulletin, 144(2), 140-176. https://doi.org/10.1037/bul0000115 


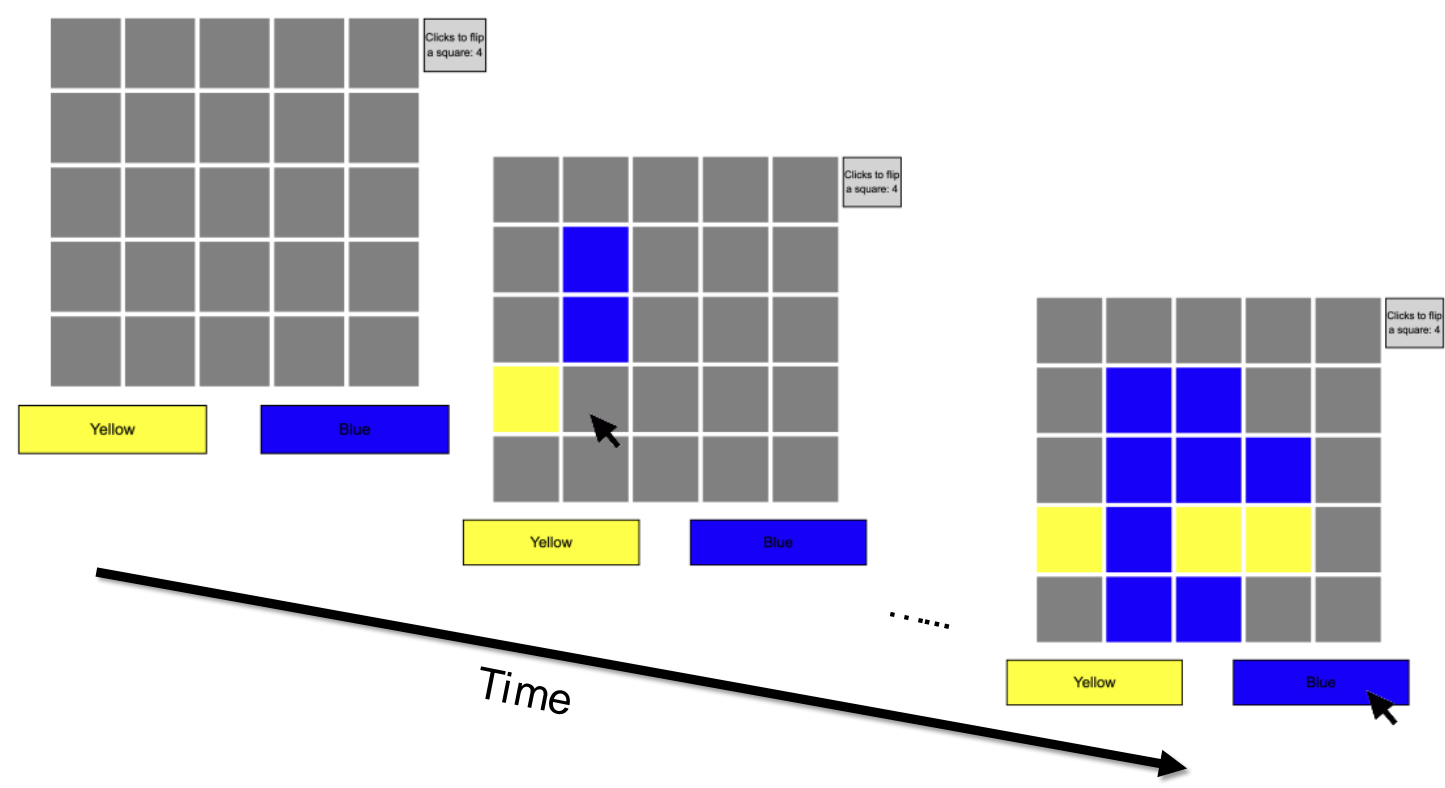

Figure 1. The effort-based information sampling task. The figure denotes a single trial in which participants iteratively sampled 11 squares prior to selecting blue as the majority underlying color. Participants flip as many squares as desired before selecting a color. The grey square in the upper right indicates the number of clicks needed to flip a square. 

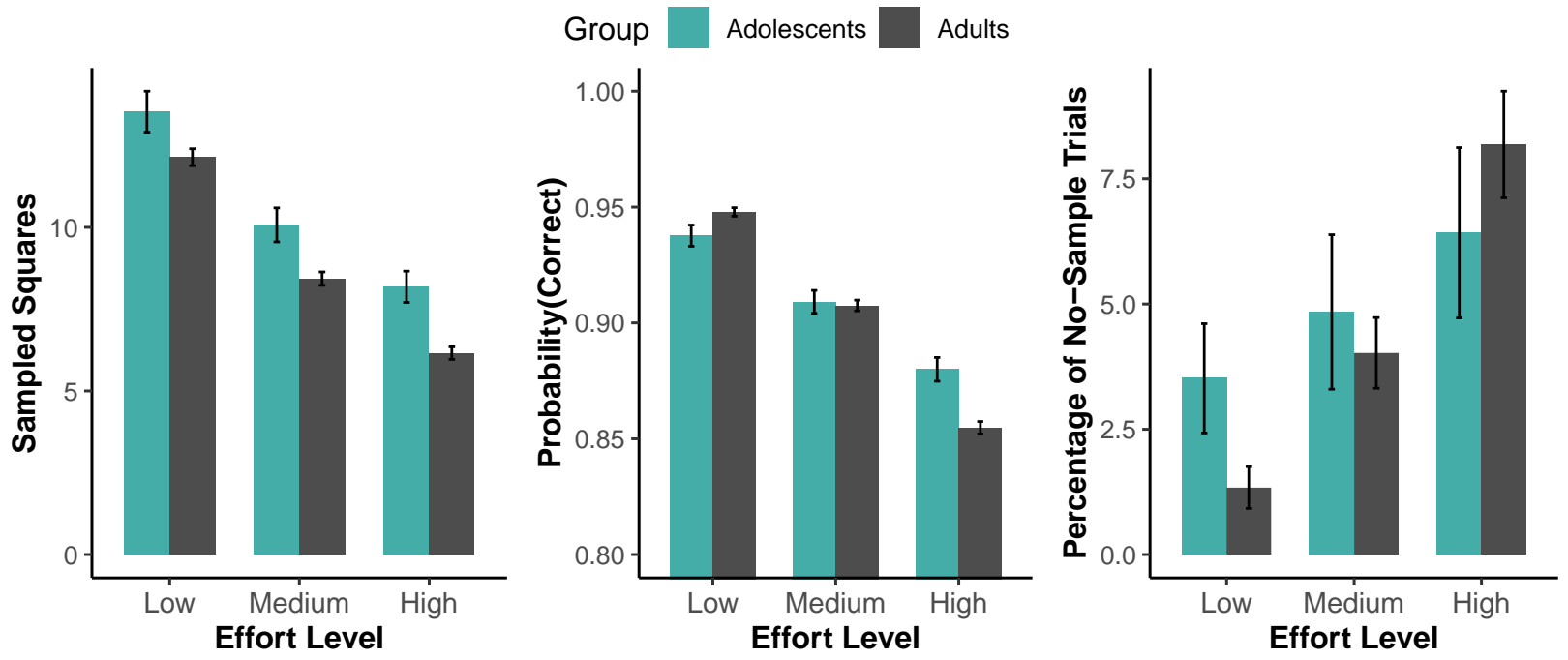

Figure 2: Left Panel: Mean number of squares sampled. Middle Panel: Mean probability of responding correctly. Right Panel: Percentage of trials without any information sampling. Bars represent the group means, and errors bars indicate standard deviation. 

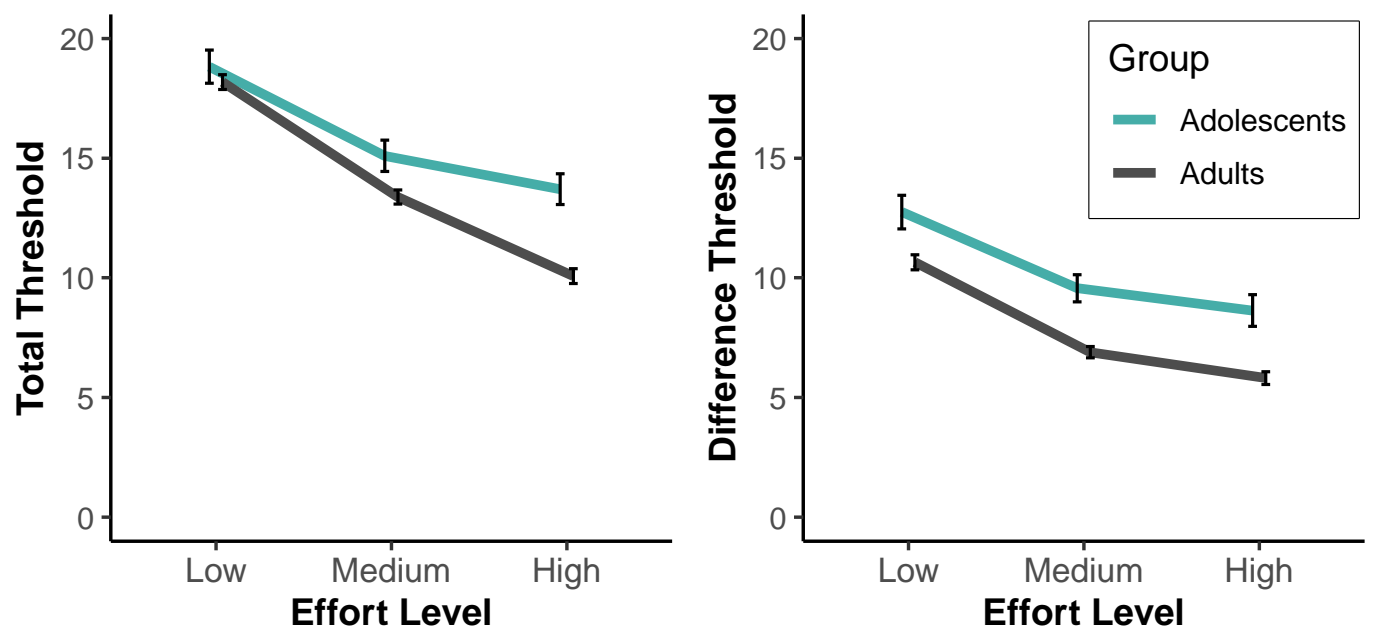

Figure 3. Best fitting parameter estimates for the two age groups for the thresholds from the Two-rule heuristic model. Group means for each threshold parameter are shown, and errors bars indicate the S.E.M. 


\begin{tabular}{l|c|c|c|c|c|c|}
\multicolumn{4}{c}{ Adolescents } & \multicolumn{3}{c}{ Adults } \\
\cline { 2 - 7 } & Low & Medium & High & Low & Medium & High \\
\hline $\begin{array}{l}\text { Squares } \\
\text { Flipped }\end{array}$ & $13.54(6.09)$ & $10.08(5.06)$ & $8.19(4.64)$ & $12.15(4.88)$ & $8.43(3.92)$ & $6.16(3.64)$ \\
\hline $\begin{array}{l}\text { p(correct) } \\
\text { No-sample } \\
\text { Trials }\end{array}$ & $0.94(0.09)$ & $0.91(0.10)$ & $0.88(0.10)$ & $0.95(0.07)$ & $0.91(0.08)$ & $0.85(0.11)$ \\
\hline
\end{tabular}

Table 1. Descriptive statistics for each age group at each effort level. Data are presented as means and standard deviations. 


\begin{tabular}{|c|c|c|c|c|c|c|c|c|}
\hline \multirow[t]{2}{*}{ Model } & \multicolumn{4}{|c|}{ Adults } & \multicolumn{4}{|c|}{ Adolescents } \\
\hline & Overall & Low & Medium & High & Overall & Low & Medium & High \\
\hline Bayes Optimal & 78048 & 42555 & 20139 & 15401 & 27652 & 14291 & 7724 & 5683 \\
\hline $\begin{array}{l}\text { Bayes Optimal } \\
\text { + Urgency }\end{array}$ & 71282 & 41016 & 17712 & 12624 & 25606 & 13313 & 7164 & 5201 \\
\hline $\begin{array}{l}\text { Bayes } \\
\text { Uncertainty }\end{array}$ & 48036 & 20688 & 15553 & 11842 & 15552 & 6234 & 4818 & 4517 \\
\hline $\begin{array}{l}\text { Bayes } \\
\text { Uncertainty + } \\
\text { Urgency }\end{array}$ & 40859 & 16444 & 13422 & 11064 & 11720 & 4140 & 3818 & 3709 \\
\hline $\begin{array}{l}\text { Absolute } \\
\text { Difference }\end{array}$ & 44791 & 17524 & 14585 & 12706 & 12910 & 4690 & 4269 & 3975 \\
\hline Total Squares & 47651 & 18589 & 15807 & 13279 & 12631 & 4516 & 4184 & 3954 \\
\hline Two-rule & 37469 & 15177 & 12141 & 10197 & 10555 & 3730 & 3562 & 3310 \\
\hline
\end{tabular}

Table 2. Bayesian Information Criterion (BIC) for each model per age group, as well as for each effort level across trials. The Two-rule model fit best for both age groups, and all effort levels. 